\title{
ASYMPTOTIC BEHAVIOR OF STABLE MANIFOLDS
}

\author{
MICHAL FEČKAN
}

(Communicated by Kenneth R. Meyer)

\begin{abstract}
The relation between local stable manifolds of an ordinary differential equation and its discretization is studied. We show that a local stable manifold of a hyperbolic fixed point of an ordinary differential equation is the limit of local stable manifolds of the same fixed point of its discretizations as the discretization parameter $h>0$ approaches 0 .
\end{abstract}

The main purpose of this paper is to investigate the following problem. Let us consider a mapping $s_{\varepsilon}: R^{n} \rightarrow R^{n}$,

$$
s_{\varepsilon}(z)=z+\varepsilon \cdot f(z)
$$

where $f \in C^{2}, \varepsilon \in R \backslash\{0\}, f(0)=0, D f(0)$ is hyperbolic, i.e., the eigenvalues of $D f(0)$ have nonzero real parts. We can write the mapping $s_{\varepsilon}$ in the form $(-)$ with $g(x, y), r(x, y)=O(|x|+|y|)$

(-) $\quad s_{\varepsilon}(x, y)=(x+\varepsilon(A x+g(x, y), y+\varepsilon(B y+r(x, y))$

where $A \in \mathscr{L}\left(R^{k}\right), B \in \mathscr{L}\left(R^{m}\right)$ have positive, negative real parts of their eigenvalues, respectively. It is easy to see that $I+\varepsilon B, I+\varepsilon A$ have no eigenvalues on the unit circle for all small $\varepsilon \neq 0$. Hence the mapping $s_{\varepsilon}$ has the hyperbolic fixed point 0 . For this point there exists a local stable manifold $W_{\varepsilon}^{s}$ for each small $\varepsilon$ [2]. The following theorem gives us information about their limit as $\varepsilon>0$ approaches 0 .

Theorem 1. There is $\delta>0$ and a continuous mapping

$$
h:\langle 0, \delta) \rightarrow C^{1}\left(B_{\delta}, R^{k}\right)
$$

such that

(i) the graph of $h(\varepsilon, \cdot) \in C^{2}\left(B_{\delta}, R^{k}\right)$ is a local stable manifold $W_{\varepsilon}^{s}$ of 0 for $s_{\varepsilon}$ and $\varepsilon>0$

(ii) the graph of $h(0, \cdot)$ is a local stable manifold of 0 for the differential equation $z^{\prime}=f(z)$

$$
\left(B_{\delta}=\left\{x \in R^{m},|x| \leq \delta\right\}\right) .
$$

Received by the editors November 21, 1989.

1980 Mathematics Subject Classification (1985 Revision). Primary 58F15. 
We remark that this problem is directly related to the method of averaging (c.f. [6] for further details).

The plan of our paper is as follows. In the first part, we present the proof of Theorem 1. In the second part, we give a simple proof of a result from [3], where the author of that paper has considered a similar problem for periodic ordinary differential equations. Finally, we investigate a discrete version of this problem.

Proof of Theorem 1. By the well-known arguments used in [1] and [5], we can suppose the existence of $\varepsilon_{0}>0, K>0$ with the properties

$$
1+\varepsilon \cdot K \leq|(I+\varepsilon \cdot A)|, \quad|I+\varepsilon \cdot B| \leq 1-\varepsilon \cdot K
$$

for each $0 \leq \varepsilon<\varepsilon_{0}$.

Let

$$
Z=\left\{\left\{\left(x_{n}, y_{n}\right)\right\}_{1}^{\infty}, x_{n} \in R^{k}, y_{n} \in R^{m}, \sup \left(\left|x_{n}\right|+\left|y_{n}\right|\right)<\infty\right\} .
$$

$Z$ is a Banach space with the norm

$$
\left|\left\{\left(x_{n}, y_{n}\right)\right\}_{1}^{\infty}\right|=\sup \left(\left|x_{n}\right|+\left|y_{n}\right|\right) .
$$

Let us consider the linear mappings

$$
\begin{array}{ll}
\bar{A}: X \rightarrow X, & \left(\bar{A}\left(\left\{x_{n}\right\}_{1}^{\infty}\right)_{n}=x_{n+1}-(I+\varepsilon A) x_{n}\right) \\
\bar{B}: \bar{X} \rightarrow \bar{X}, & \left(\bar{B}\left(\left\{y_{n}\right\}_{1}^{\infty}\right)_{n}=y_{n+1}-(I+\varepsilon B) y_{n}\right)
\end{array}
$$

where

$$
\begin{aligned}
& X=\left\{\left\{x_{n}\right\}_{1}^{\infty}, x_{n} \in R^{k}, \sup \left|x_{n}\right|<\infty\right\} \\
& \bar{X}=\left\{\left\{y_{n}\right\}_{1}^{\infty}, y_{n} \in R^{m}, \sup \left|y_{n}\right|<\infty\right\}
\end{aligned}
$$

We solve the equation

$$
x_{n+1}-(I+\varepsilon A) x_{n}=h_{n}
$$

in the space $X$. This equation has a unique bounded solution for all small $\varepsilon>0$ [7, p. 272] (i.e., $1 \gg \varepsilon>0$ ), namely,

$$
x_{n}=-\left((I+\varepsilon A)^{-1} h_{n}+(I+\varepsilon A)^{-2} h_{n+1}+\cdots\right), \quad n \geq 1 .
$$

Hence

$$
\begin{aligned}
\left|x_{n}\right| & \leq \frac{\left|h_{n}\right|}{(1+\varepsilon \cdot K)}+\frac{\left|h_{n+1}\right|}{(1+\varepsilon \cdot K)^{2}}+\cdots \\
& \leq|h| \cdot\left(\frac{1}{1+K \varepsilon}+\cdots\right)=|h| \cdot \frac{1}{\varepsilon \cdot K}
\end{aligned}
$$

Thus

$$
|x| \leq|h| \cdot \frac{1}{\varepsilon \cdot K}, \quad x=\left\{x_{n}\right\}_{1}^{\infty}, \quad h=\left\{h_{n}\right\}_{1}^{\infty} .
$$

In the same way we solve in $\bar{X}$

$$
y_{n+1}-(I+\varepsilon B) y_{n}=h_{n} .
$$


This equation has a unique bounded solution for $1 \gg \varepsilon>0$ with the initial condition $y_{1}=c$ :

$$
y_{n}=(I+\varepsilon B)^{n-1} c+(I+\varepsilon B)^{n-2} h_{1}+\cdots+h_{n-1}, \quad n \geq 2 .
$$

Hence

$$
\begin{aligned}
\left|y_{n}\right| & \leq|c|+|h| \cdot\left((1-\varepsilon \cdot K)^{n-2}+\cdots+1\right) \\
& <|c|+|h| \frac{1}{\varepsilon \cdot K} .
\end{aligned}
$$

Finally, we solve the equation

$$
\begin{aligned}
& x_{n+1}=(I+\varepsilon A) x_{n}+\varepsilon g\left(x_{n}, y_{n}\right) \\
& y_{n+1}=(I+\varepsilon B) y_{n}+\varepsilon r\left(x_{n}, y_{n}\right)
\end{aligned}
$$

in $Z$ near $(0,0)$. By the above results (1) can be written in the form

$$
\begin{aligned}
& \left\{x_{n}\right\}_{1}^{\infty}=\varepsilon(\bar{A})^{-1}\left(\left\{g\left(x_{n}, y_{n}\right)\right\}_{1}^{\infty}\right) \\
& \left\{y_{n}\right\}_{2}^{\infty}=\varepsilon(\bar{B})^{-1}\left(\left\{r\left(x_{n}, y_{n}\right)\right\}_{1}^{\infty}\right), \quad y_{1}=c .
\end{aligned}
$$

But $\varepsilon(\bar{A})^{-1}, \varepsilon(\bar{B})^{-1}$ are uniformly bounded for $1 \gg \varepsilon>0$. Hence we can uniformly apply the implicit function theorem to obtain a solution of $(+)$ :

$$
\left\{x_{n}(\varepsilon, c)\right\}_{1}^{\infty}, \quad\left\{y_{n}(\varepsilon, c)\right\}_{2}^{\infty} .
$$

From this it follows that the graph $W_{\varepsilon}^{s}$ of $x_{1}(\varepsilon, \cdot)$ in $B_{\delta} \times R^{k}$ contains all points from which bounded orbits of $s_{\varepsilon}$ near $0 \in R^{k} \times R^{m}$ start. Obviously $x_{1}(\varepsilon, \cdot) \in C^{2}\left(B_{\delta}, R^{k}\right)$ and the set

$$
\left\{x_{1}(\varepsilon, \cdot)\right\}_{\varepsilon \in\left(0, \varepsilon_{0}\right)}
$$

is bounded in $C^{2}\left(B_{\delta}, R^{k}\right)$.

Now we show that $W_{\varepsilon}^{s}$ is the local stable manifold of 0 for $s_{\varepsilon}$. By (1) we have

$$
\begin{aligned}
& \left|x_{n}\right| \leq\left|(I+\varepsilon A)^{-1}\right| \cdot\left|h_{n}\right|+\left|(I+\varepsilon A)^{-2}\right| \cdot\left|h_{n+1}\right|+\cdots, \quad h_{n}=\varepsilon \cdot g\left(x_{n}, y_{n}\right) \\
& \left|y_{n}\right| \leq\left|(I+\varepsilon B)^{n-1}\right| \cdot|c|+\left|(I+\varepsilon B)^{n-2}\right| \cdot\left|g_{1}\right|+\cdots+\left|g_{n-1}\right|, \quad g_{n}=\varepsilon \cdot r\left(x_{n}, y_{n}\right) .
\end{aligned}
$$

If $\overline{\lim }\left(\left|x_{n}\right|+\left|y_{n}\right|\right)=b>0$ for a small $\left\{\left(x_{n}, y_{n}\right)\right\}_{1}^{\infty}$ then

$$
\left|h_{n}\right| \leq \frac{K}{3} \cdot b \cdot \varepsilon, \quad\left|g_{n}\right| \leq \frac{K}{3} \cdot b \cdot \varepsilon, n \gg 1 \text { (i.e., } n \text { is large). }
$$

Hence

$$
\begin{aligned}
\left|x_{n}\right| & \leq \frac{1}{1+\varepsilon \cdot K} \cdot \frac{K}{3} \cdot b \cdot \varepsilon+\frac{1}{(1+\varepsilon \cdot K)^{2}} \cdot \frac{K}{3} \cdot b \cdot \varepsilon+\cdots \\
& =\frac{1}{3} \cdot b, \quad n \gg 1
\end{aligned}
$$


and for a fixed large $p$

$$
\begin{aligned}
\left|y_{n}\right| \leq & (1-\varepsilon K)^{n-1} \cdot|c|+\cdots+(1-\varepsilon K)^{n-p} \cdot\left|g_{p-1}\right| \\
& +(1-\varepsilon K)^{n-p-1} \cdot \frac{K \varepsilon}{3} \cdot b+\cdots+\frac{K \varepsilon}{3} \cdot b
\end{aligned}
$$

We see that

$$
\overline{\lim }\left(\left|x_{n}\right|+\left|y_{n}\right|\right) \leq \frac{1}{3} \cdot b+\frac{1}{3} \cdot b<b .
$$

This proves that $\lim \left(\left|x_{n}\right|+\left|y_{n}\right|\right)=0$.

Now we take a sequence $\left\{x_{1}\left(\varepsilon_{n}, \cdot\right)\right\}_{n=1}^{\infty} \in C^{2}\left(B_{\delta}, R^{k}\right)$ such that $\varepsilon_{n}>0$, $\varepsilon_{n} \rightarrow 0$. This sequence is bounded in $C^{2}\left(B_{\delta}, R^{k}\right)$ and hence there exists a subsequence $\left\{x_{1}\left(\varepsilon_{n_{i}}, \cdot\right)\right\}_{i=1}^{\infty}$ which has a limit point $h \in C^{1}\left(B_{\delta}, R^{k}\right)$ in the space $C^{1}\left(B_{\delta}, R^{k}\right)$.

On the other hand, since $W_{\varepsilon}^{s}$ is the invariant manifold of $s_{\varepsilon}, x_{1}(\varepsilon, \cdot)$ must satisfy

$$
(I+\varepsilon A) x_{1}(\varepsilon, y)+\varepsilon g\left(x_{1}(\varepsilon, y), y\right)=x_{1}\left(\varepsilon,(I+\varepsilon B) y+\varepsilon r\left(x_{1}(\varepsilon, y), y\right)\right) .
$$

Hence

$$
\begin{aligned}
& \varepsilon_{n_{i}}\left(A x_{1}\left(\varepsilon_{n_{i}}, y\right)+g\left(x_{1}\left(\varepsilon_{n_{i}}, y\right), y\right)\right) \\
& \quad=x_{1}\left(\varepsilon_{n_{i}},\left(I+\varepsilon_{n_{i}} B\right) y+\varepsilon_{n_{i}} r\left(x_{1}\left(\varepsilon_{n_{i}}, y\right), y\right)\right)-x_{1}\left(\varepsilon_{n_{i}}, y\right)
\end{aligned}
$$

and

$$
\begin{aligned}
& A x_{1}\left(\varepsilon_{n_{i}}, y\right)+g\left(x_{1}\left(\varepsilon_{n_{i}}, y\right), y\right) \\
& \quad=\left(D_{y} x_{1}\left(\varepsilon_{n_{i}}, y\right)+O\left(\varepsilon_{n_{i}}\right)\right)\left(B y+r\left(x_{1}\left(\varepsilon_{n_{i}}, y\right), y\right)\right)
\end{aligned}
$$

where we used the mean value theorem. Hence

$$
\begin{gathered}
A h(y)+g(h(y), y)=D_{y} h(y)(B y+r(h(y), y)) \\
h(0)=0 .
\end{gathered}
$$

If $\delta$ is sufficiently small, then (4) has a unique solution and the graph of $h$ is a local stable manifold of 0 for the equation

$$
z^{\prime}=f(z) \text {. }
$$

Indeed, the point 0 is hyperbolic for $z^{\prime}=f(z)$. By the assumptions on $f$ the point 0 has local stable and local unstable $C^{2}$-manifolds. Using a $C^{2}$-change of coordinates we can consider that the $x$-axis is the local unstable manifold and the $y$-axis is the local stable manifold. In these new coordinates the equation $z^{\prime}=f(z)$ has the form $z^{\prime}=f_{1}(z)$, i.e.,

$$
\begin{aligned}
x^{\prime} & =A_{1} x+g_{1}(x, y) \\
y^{\prime} & =B_{1} y+r_{1}(x, y)
\end{aligned}
$$


where $A_{1}, B_{1}$ have the above properties, $g_{1}(x, y)=O(|x|) \cdot O(|x|+|y|)$, $r_{1}(x, y)=O(|y|) \cdot O(|x|+|y|)$. We can assume that

$$
\left(B_{1} y, y\right) \leq-a \cdot|y|^{2}, \quad a>0
$$

where $(\cdot, \cdot)$ is a scalar product. The graph of $h$ in the new coordinates is the graph of some mapping $h_{1}: B_{\delta} \rightarrow R^{k}$. We have for a small $|x|+|y|$ :

$$
\left(B_{1} y+r_{1}(x, y), y\right) \leq-a_{1} \cdot|y|^{2}, \quad 0<a_{1}<a .
$$

Using this property and the fact that the graph of $h_{1}$ is invariant by $z^{\prime}=f_{1}(z)$, we see that the graph of $h$ is the local stable manifold of 0 for $z^{\prime}=f(z)$.

From the above results we obtain the proof of Theorem 1 .

We note that using the above method we can give a simple proof of a result from the paper [3]. The author of that paper considers an ordinary differential equation

$$
z^{\prime}=\varepsilon f(z, t, \varepsilon)
$$

where by [3, Proposition 2.2] we can suppose that

$$
f(z, t, \varepsilon)=(A x+g(x, y, t, \varepsilon), B y+r(x, y, t, \varepsilon))
$$

where $A, B$ have the above properties, $r, g$ are $2 \pi$-periodic in $t, r, g \in C^{2}$, $g(0,0, \cdot, \cdot)=0, D_{x, y} g(0,0, \cdot, 0)=0, r(0,0, \cdot, \cdot)=0, D_{x, y} r(0,0, \cdot, 0)=$ 0 .

The appropriate spaces are the following:

$$
\begin{aligned}
& Z=\left\{(x, y), x \in C^{0}\left(R_{+}, R^{k}\right), y \in C^{0}\left(R_{+}, R^{m}\right), \sup (|x|+|y|)<\infty\right\} \\
& X=\left\{x \in C^{0}\left(R_{+}, R^{k}\right), \sup |x|<\infty\right\} \\
& \bar{X}=\left\{y \in C^{0}\left(R_{+}, R^{m}\right), \sup |y|<\infty\right\} .
\end{aligned}
$$

By the variation of the constants formula we can obtain an equation similar to $(+)$. Solving this equation we have a mapping

$$
h(\varepsilon, \cdot, \cdot) \in C^{2}\left(B_{\delta} \times R, R^{k}\right)
$$

where $(h(\varepsilon, y, t), y)$ is the initial value from which a bounded orbit of (6) near $0 \in R^{k} \times R^{m}$ starts. Moreover, the set

$$
\{h(\varepsilon, \cdot, \cdot)\}_{\varepsilon \in\left(0, \varepsilon_{0}\right)}
$$

is bounded in $C^{2}\left(B_{\delta} \times R, R^{k}\right)$ and $h(\varepsilon, \cdot, \cdot)$ is $2 \pi$-periodic in $t$. A result of Hale [4, pp. 166-167] gives us that the graph $W_{\varepsilon}^{s}$ of $h(\varepsilon, \cdot, \cdot)$ is a local stable manifold of 0 for (6). Hence $W_{\varepsilon}^{s}$ is invariant by (6) and this implies:

$$
D_{t} h(\varepsilon, \cdot, \cdot)+D_{y} h(\varepsilon, \cdot, \cdot)(\varepsilon B \cdot+\varepsilon r(h, \cdot, \cdot, \varepsilon))=\varepsilon A h+\varepsilon g(h, \cdot, \cdot, \varepsilon) .
$$

Since

$$
\frac{1}{2 \pi} \int_{0}^{2 \pi} D_{t} h(\cdot, \cdot, s) d s=0
$$


we obtain

$$
\begin{aligned}
& \frac{1}{2 \pi} \int_{0}^{2 \pi} D_{y} h(\varepsilon, \cdot, s)(B \cdot+r(h, \cdot, s, \varepsilon)) d s \\
& \quad=\frac{1}{2 \pi} \int_{0}^{2 \pi}(A h(\varepsilon, \cdot, s)+g(h, \cdot s, \varepsilon) d s .
\end{aligned}
$$

We take a sequence $\left\{h\left(\varepsilon_{n}, \cdot, \cdot\right)\right\}_{1}^{\infty}, \varepsilon_{n} \rightarrow 0$. Then there is a subsequence $\left\{h\left(\varepsilon_{n_{i}}, \cdot, \cdot\right)\right\}_{1}^{\infty}$ which tends to $\bar{h} \in C^{1}\left(B_{\delta} \times R, R^{k}\right)$ in this space. Hence

$$
\begin{gathered}
\frac{1}{2 \pi} \int_{0}^{2 \pi} D_{y} \bar{h}(\cdot, s)\left(B \cdot+r(\bar{h}, \cdot, s, 0) d s=\frac{1}{2 \pi} \int_{0}^{2 \pi}(A \bar{h}(\cdot, s)+g(\bar{h}, \cdot, s, 0)) d s\right. \\
D_{t} \bar{h}(\cdot, s)=0 .
\end{gathered}
$$

We see that $\bar{h}$ is independent of $t, \bar{h}(y, t)=\bar{h}(y)$ and

$$
\begin{gathered}
D_{y} \bar{h} \cdot\left(B .+\frac{1}{2 \pi} \int_{0}^{2 \pi} r(\bar{h}, \cdot, s, 0) d s\right)=A \bar{h}+\frac{1}{2 \pi} \int_{0}^{2 \pi} g(\bar{h}, \cdot, s, 0) d s \\
\bar{h}(0)=0 .
\end{gathered}
$$

This gives us that the graph of $\bar{h}$ is a local stable manifold of 0 of the equation

$$
z^{\prime}=\frac{1}{2 \pi} \int_{0}^{2 \pi} f(z, s, 0) d s .
$$

Summing up we have [3]:

Theorem 2. There exists $\delta>0$ and $a C^{0}$-mapping

$$
h:\langle 0, \delta) \rightarrow C^{1}\left(B_{\delta} \times R, R^{k}\right)
$$

such that

(i) $h$ is $2 \pi$-periodic in $t$,

(ii) the graph of $h(\varepsilon, \cdot, \cdot) \in C^{2}\left(B_{\delta} \times R, R^{k}\right)$ in $R^{n} \times R$ is a local stable manifold of 0 of (6) for $\varepsilon>0$,

(iii) the graph of $h(0, \cdot, \cdot)$ in $R^{n} \times R$ is $W \times R$, where $W$ is a local stable manifold of 0 for (7).

Finally, we shall investigate a discrete version of (6). For each initial point $(x, m) \in R^{n} \times Z$, where the set $Z$ is the set of integers, we define an orbit $\left\{x_{i}\right\}_{m}^{\infty}$ in the following way

$$
x_{i+1}=x_{i}+\varepsilon g\left(x_{i}, i, \varepsilon\right), \quad i \geq m, x_{m}=x
$$

where $g \in C^{2}, g: R^{n} \times Z \times R \rightarrow R^{n}$ and $g$ is $p$-periodic in the second variable, i.e., $g(\cdot, i+p, \cdot)=g(\cdot, i, \cdot)$.

Consider also the averaged mapping

$$
\bar{g}(x)=\frac{1}{p} \sum_{1}^{p} g(x, i, 0) .
$$

Suppose $\bar{g}(0)=0$ and $D \bar{g}(0)$ is hyperbolic, i.e., has no eigenvalues on the imaginary axis. 
Lemma 3. There exists $\varepsilon_{1}>0$ and a $C^{2}$-mapping

$$
x:\{1, \ldots, p\} \times\left(-\varepsilon_{1}, \varepsilon_{1}\right) \rightarrow R^{n}
$$

such that for each $\varepsilon \in\left(-\varepsilon_{1}, \varepsilon_{1}\right), \varepsilon \neq 0$ (8) has a unique small p-periodic orbit $\{x(i, \varepsilon)\}_{i=1}^{p}$ and $x(\cdot, 0)=0$.

Proof. We solve the equation

$$
\begin{aligned}
x_{2}= & x_{1}+\varepsilon g\left(x_{1}, 1, \varepsilon\right) \\
& \cdot \\
& \cdot \\
& \cdot \\
& \cdot \\
x_{p}= & x_{p-1}+\varepsilon g\left(x_{p-1}, p-1, \varepsilon\right) \\
x_{1}= & x_{p}+\varepsilon g\left(x_{p}, p, \varepsilon\right) .
\end{aligned}
$$

Setting

$$
\begin{aligned}
w & =\left(x_{1}, \ldots, x_{p}\right), \quad H w=\left(x_{2}-x_{1}, \ldots, x_{1}-x_{p}\right) \\
F_{\varepsilon}(w) & =\left(g\left(x_{1}, 1, \varepsilon\right), \ldots, g\left(x_{p}, p, \varepsilon\right)\right) \\
P w & =\left(\frac{x_{1}+\cdots+x_{p}}{p}, \ldots, \frac{x_{1}+\cdots+x_{p}}{p}\right)
\end{aligned}
$$

this equation can be rewritten in the form

$$
\begin{aligned}
H u & =(I-P) \cdot \varepsilon F_{\varepsilon}(u+v), \quad u \in \operatorname{Im} H \\
0 & =\varepsilon P F_{\varepsilon}(u+v), \quad v \in \operatorname{Ker} H,
\end{aligned}
$$

since $\left(R^{n}\right)^{p}=\operatorname{Ker} H \oplus \operatorname{Im} H$ and $\operatorname{Ker} P=\operatorname{Im} H, \operatorname{Im} P=\operatorname{Ker} H$. Now using the implicit function theorem the first equation has a solution $u(\varepsilon, v)$ for $\varepsilon$, $v$ small such that $u(0, \cdot)=0$. Hence

$$
0=P F_{\varepsilon}(u(\varepsilon, v)+v) \text {. }
$$

We can also apply the implicit function theorem for this equation since $v \in$ $\operatorname{Ker} H=\left\{x \in\left(R^{n}\right)^{p}, x_{1}=\cdots=x_{p}=z\right\}, P F_{0}(u(0, v)+v)=(\bar{g}(z), \cdots, \bar{g}(z))$ and $\bar{g}(0)=0, D \bar{g}(0)$ is invertible.

From Lemma 3 we make the $p$-periodic coordinate change

$$
z_{i}=x_{i}-x(i, \varepsilon)
$$

and (8) becomes

$$
z_{i+1}=z_{i}+\varepsilon f\left(z_{i}, i, \varepsilon\right)
$$

where $f \in C^{2}, f(0, i, \varepsilon)=0$ and $\bar{g}(y)=\bar{f}(y)=\frac{1}{p} \sum_{1}^{p} f(y, i, 0)$. Next we use averaging to make (9) autonomous to order 1 . 
Lemma 4. Let $f$ be as in (9). Then by the change of coordinates

$$
\begin{gathered}
y_{i}=z_{i}+\varepsilon \sum_{j=1}^{i-1}\left(f\left(z_{i}, j, 0\right)-\bar{g}\left(z_{i}\right)\right), \quad i>1 \\
y_{1}=z_{1}
\end{gathered}
$$

(9) becomes

$$
y_{i+1}=y_{i}+\varepsilon h\left(y_{i}, i, \varepsilon\right)
$$

where $h \in C^{2}, h(x, i, 0)=\bar{f}(x)$.

Proof. Using the above change of coordinates we immediately obtain the assertion of Lemma 4 . We note that this change of coordinates is $p$-periodic in the second variable.

Hence our problem (8) can be reduced to (10), where $\bar{f}(x)=\bar{g}(x)$ has the hyperbolic fixed point $x=0$. Next we write (10) in the following form

$$
\begin{aligned}
& x_{i+1}=x_{i}+\varepsilon\left(A x_{i}+s\left(x_{i}, y_{i}, i, \varepsilon\right)\right), \\
& y_{i+1}=y_{i}+\varepsilon\left(B y_{i}+r\left(x_{i}, y_{i}, i, \varepsilon\right)\right), \quad i \geq m
\end{aligned}
$$

where $A, B$ have the properties from Theorem 1: $s, r \in C^{2}, s(0,0, i, \varepsilon)=$ $0, r(0,0, i, \varepsilon)=0, D_{x, y} s(0,0, \cdot, 0)=0, D_{x, y} r(0,0, \cdot, 0)=0$ and $s$, $r$ are $p$-periodic in $i$. Now we can use the above procedure to obtain $C^{2}$ mappings $h_{m}(\cdot, \varepsilon) \in C^{2}\left(B_{\delta}, R^{k}\right)$ such that the graph $W_{\varepsilon}^{m}$ of $h_{m}$ is a local stable manifold of $0 \in R^{n}$ of (11). We note that iterations in (11) start from $i=m$. By the periodic condition for $r, s$ we have

$$
h_{p+i}=h_{i} \text {. }
$$

In this case the set $\left\{h_{m}(\cdot, \varepsilon)\right\}_{\varepsilon \in\left(0, \varepsilon_{0}\right)}$ is also bounded in $C^{2}\left(B_{\delta}, R^{k}\right)$. We take a sequence $\left\{\left(h_{1}\left(\cdot, \varepsilon_{i}\right), \ldots, h_{p}\left(\cdot, \varepsilon_{i}\right)\right)\right\}_{i=1}^{\infty}, \varepsilon_{i} \rightarrow 0$. Then there exists a subsequence $\left\{\left(h_{1}\left(\cdot, \varepsilon_{n_{i}}\right), \ldots, h_{p}\left(\cdot, \varepsilon_{n_{i}}\right)\right)\right\}_{i=1}^{\infty}$ which has a limit point $\left(h_{1}, \ldots, h_{p}\right)$ in the space

$$
C^{1}\left(B_{\delta}, R^{k}\right) \times \cdots \times C^{1}\left(B_{\delta}, R^{k}\right) .
$$

(This follows from the well-known theorem of Arzela-Ascoli and we also used this theorem in the above proofs of our theorems.) Since the sequence $\left\{W_{\varepsilon}^{1}\right.$, $\left.\ldots, W_{\varepsilon}^{p}\right\}$ is invariant for (11) we have

$$
\begin{aligned}
& h_{i+1}\left(y+\varepsilon\left(B y+r\left(h_{i}(x, \varepsilon), y, i, \varepsilon\right)\right), \varepsilon\right) \\
& \quad=h_{i}(y, \varepsilon)+\varepsilon\left(A h_{i}(y, \varepsilon)+s\left(h_{i}(y, \varepsilon), y, i, \varepsilon\right)\right) .
\end{aligned}
$$

Thus by the mean value theorem

$$
\begin{aligned}
& \left(D_{y} h_{i+1}(y, \varepsilon)+O(\varepsilon)\right) \cdot\left(B y+r\left(h_{i}(y, \varepsilon), y, i, \varepsilon\right)\right) \\
& \quad=\varepsilon\left(A h_{i}(y, \varepsilon)+s\left(h_{i}(y, \varepsilon), y, i, \varepsilon\right)\right)+h_{i}(y, \varepsilon)-h_{i+1}(y, \varepsilon)
\end{aligned}
$$


and

$$
\begin{gathered}
\frac{1}{p} \sum_{i=1}^{p}\left(D_{y} h_{i+1}(y, \varepsilon)+O(\varepsilon)\right)\left(B y+r\left(h_{i}(y, \varepsilon), y, i, \varepsilon\right)\right) \\
=\frac{1}{p} \sum_{i=1}^{p}\left(A h_{i}(y, \varepsilon)+s\left(h_{i}(y, \varepsilon), y, i, \varepsilon\right)\right) .
\end{gathered}
$$

Hence we obtain

$$
h_{1}=h_{2}=\cdots=h_{p}=h
$$

and

$$
\begin{gathered}
\frac{1}{p} \sum_{i=1}^{p} D_{y} h(y)(B y+r(h(y), y, i, 0)) \\
=\frac{1}{p} \sum_{i=1}^{p}(A h(y)+s(h(y), y, i, 0)) \\
h(0)=0 .
\end{gathered}
$$

Thus the graph of $h$ is a local stable manifold for the averaged equation

$$
z^{\prime}=\bar{g}(z) \text {. }
$$

From the above results we obtain the following theorem.

Theorem 5. There exists $\delta>0$ and a $C^{0}$-mapping

$$
h:\{1,2, \ldots, p\} \times\langle 0, \delta) \rightarrow C^{1}\left(B_{\delta}, R^{k}\right)
$$

such that

(1) The graph of $h(\cdot, \cdot, \varepsilon), \varepsilon>0$ is a local stable manifold of a unique small p-periodic orbit of (8).

(2) There is a local stable manifold $W$ of 0 of the averaged equation (12) such that the graph of $h(\cdot, \cdot, 0)$ is $W \times\{1,2, \ldots, p\}$. (Note that the stable manifolds are subsets of $R^{n} \times\{1,2, \ldots, p\}$.)

\section{REFERENCES}

1. M. W. Hirsch and S. Smale, Differential equations, dynamical systems and linear algebra, Academic Press, New York and London, 1974.

2. S. N. Chow and J. K. Hale, Methods of bifurcation theory, Springer-Verlag, New York, 1983.

3. S. Schecter, Stable manifolds in the method of averaging, Trans. Amer. Math. Soc. 308 (1988), 159-176.

4. J. K. Hale, Ordinary differential equations, Krieger, Huntington, New York, 1980.

5. M. Medved, Dynamical systems, Veda, Bratislava, 1988. (Slovak)

6. J. Murdock and C. Robinson, Qualitative dynamics from asymptotic expansions: local theory, J. Differential Equations 36 (1980), 425-441.

7. K. J. Palmer, Exponential dichotomies, the shadowing lemma and transversal homoclinic points, Dynamics Reported 1, Wiley, New York and Teubner, Stuttgart, 1988, pp. 265306.

Mathematical Institute of the Slovak Academy of Sciences, Bratislava, CzechosloVAKIA 